\title{
Erratum to: Chronic Kidney Disease Japan Cohort study: baseline characteristics and factors associated with causative diseases and renal function
}

\author{
Enyu Imai - Seiichi Matsuo $\cdot$ Hirofumi Makino • \\ Tsuyoshi Watanabe $\cdot$ Tadao Akizawa $\cdot$ Kosaku Nitta \\ Satoshi Iimuro · Yasuo Ohashi · Akira Hishida
}

Published online: 6 October 2010

(C) Japanese Society of Nephrology 2010

\section{Erratum to: Clin Exp Nephrol \\ DOI 10.1007/s10157-010-0328-6}

In the Appendix, the name of Daijo Inaguma was misspelled in the original version as Daijyo Inaguma.

In Tables 1 and 4, the numbers given for Creatinine clearance values were incorrect. The correct values are indicated on the following page.

For The CKD-JAC Study Group.

The online version of the original article can be found under doi: 10.1007/s10157-010-0328-6.

\section{E. Imai $(\bowtie) \cdot$ S. Matsuo}

Department of Nephrology, Nagoya University Graduate School of Medicine, 65 Tsurumai, Showa-ku, Nagoya 466-8550, Japan e-mail: imai@med.nagoya-u.ac.jp

\section{H. Makino}

Department of Medicine and Clinical Science,

Okayama University Graduate School of Medicine,

Dentistry and Pharmaceutical Sciences, Okayama, Japan

T. Watanabe

The Third Department of Internal Medicine,

Fukushima Medical College, Fukushima, Japan

T. Akizawa

Department of Nephrology, Showa University, Tokyo, Japan

K. Nitta

Department of Medicine, Kidney Center,

Tokyo Women's Medical University, Tokyo, Japan

S. Iimuro · Y. Ohashi

Department of Biostatistics, School of Public Health,

The University of Tokyo, Tokyo, Japan

A. Hishida

First Department of Medicine, Hamamatsu University School

of Medicine, Hamamatsu, Japan 
Table 1 Patient characteristics classified by causative disease

\begin{tabular}{lllllll}
\hline Variable & $\begin{array}{l}\text { Cohort, } \\
N=2977\end{array}$ & No diabetes & & Diabetes & $P$ value \\
& & $\begin{array}{l}\text { No CGN, } \\
N=909\end{array}$ & $\begin{array}{l}\text { CGN, } \\
N=948\end{array}$ & & $\begin{array}{l}\text { No nephropathy, } \\
N=507\end{array}$ & $\begin{array}{l}\text { Nephropathy, } \\
N=613\end{array}$ \\
\hline Ccr (ml/min) & & & & & \\
Mean (SD) & $48.03(29.98)$ & $44.31(26.34)$ & $49.12(30.05)$ & & $51.36(32.83)$ & $48.74(32.20)$ \\
Median (max-min) & $41.70(4.8-240.0)$ & $39.62(7.0-151.7)$ & $44.10(4.8-240.0)$ & $42.95(10.7-172.5)$ & $41.80(11.7-180.3)$ \\
1Q-3Q & $26.90-59.50$ & $24.80-57.50$ & $27.80-59.70$ & & $29.30-59.69$ & $24.50-62.10$ \\
\hline
\end{tabular}

Table 4 Baseline characterization

\begin{tabular}{|c|c|c|c|c|c|}
\hline & $\begin{array}{l}\text { Stage } 3 \mathrm{~A} \\
\text { GFR } \geq 45 \\
N=304\end{array}$ & $\begin{array}{l}\text { Stage } 3 \mathrm{~B} \\
45>\mathrm{GFR} \geq 30 \\
N=1037\end{array}$ & $\begin{array}{l}\text { Stage } 4 \\
30>\mathrm{GFR} \geq 15 \\
N=1160\end{array}$ & $\begin{array}{l}\text { Stage } 5 \\
\text { GFR }<15 \\
N=476\end{array}$ & $P$ value \\
\hline \multicolumn{6}{|l|}{$\operatorname{Ccr}(\mathrm{ml} / \mathrm{min})$} \\
\hline Mean (SD) & 90.65 (34.77) & $62.88(27.75)$ & 37.78 (15.37) & $20.92(9.11)$ & $<0.0001$ \\
\hline Median (min-max) & $82.05(30.9-180.3)$ & $56.40(8.8-240.0)$ & $34.31(7.2-97.7)$ & $19.30(4.8-53.8)$ & \\
\hline 1Q-3Q & $67.25-114.09$ & $45.20-71.20$ & $26.85-46.10$ & $14.81-25.09$ & \\
\hline
\end{tabular}

